\title{
Evaluación de la temperatura y concentración de dos agentes osmodeshidratantes en la obtención de Vaccinium myrtillus "arándano" deshidratado
}

\section{Evaluation of the temperature and concentration of two osmodeshydratants agents in obtaining Vaccinium myrtillus "blueberry" dehydrated}

\author{
Genri Hitalo Soto Medina ${ }^{1}$, Yuri Guablocho Chávez ${ }^{2}$ *Segundo Víctor Olivares Nuñoz ${ }^{3}$
}

\begin{abstract}
RESUMEN
La investigación tuvo por objetivo evaluar la temperatura y concentración de dos agentes osmodeshidratantes en la obtención de Vaccinium myrtillus "arándano" deshidratado. Bajo un Diseño Completo al Azar, se sometió el arándano fresco en frutos enteros a osmodeshidratación, en jarabe de sacarosa y miel de abeja a concentraciones 60 y $70^{\circ}$ Brix y temperaturas de 50 y $60^{\circ} \mathrm{C}$, con una relación jarabe $3: 1$, luego se secó con aire a $60^{\circ} \mathrm{C}$ y $3,5 \mathrm{~m} / \mathrm{s}$ en un secador de bandejas. Se evaluó sólidos totales, cenizas, humedad, acidez total, índice de madurez, ácido ascórbico, $\mathrm{pH}$, pérdida de peso, agua y ganancia de sólidos; también se evaluó el grado de aceptación mediante evaluación sensorial. El tratamiento con sacarosa $\left(70^{\circ}\right.$ Brix y $\left.50^{\circ} \mathrm{C}\right)(\mathrm{T} 3)$ tuvo mayor aceptación, con pérdida de peso de $29,70 \%$, pérdida de agua de $34,4 \%$; ganancia de sólidos de $6,83 \%$ y contenido de ácido ascórbico de $11,3 \mathrm{mg} / 100 \mathrm{~g}$; cuando se empleó miel de abeja a $70^{\circ}$ Brix y $60^{\circ} \mathrm{C}$, se obtuvo la mayor ganancia de sólidos; la mayor pérdida de agua se obtuvo con miel de abeja $\left(60\right.$ y $70^{\circ} \mathrm{Brix}$ a $\left.50^{\circ} \mathrm{C}\right)$.
\end{abstract}

Palabras clave: osmodeshidratación, Vaccinium myrtillus, sacarosa, miel de abeja

\begin{abstract}
The objective of the research was to evaluate the temperature and concentration of two osmohydrating agents in the production of dehydrated Vaccinium myrtillus. Under a Complete Random Design, fresh cranberry in whole fruits was subjected to osmodeshydration, in sucrose syrup and honey at concentrations 60 and $70^{\circ}$ Brix and temperatures of 50 and $60^{\circ} \mathrm{C}$, with a syrup ratio of $3: 1$, then dried with air at $60^{\circ} \mathrm{C}$ and $3.5 \mathrm{~m} / \mathrm{s}$ in a tray dryer. Total solids, ash, moisture, total acidity, maturity index, ascorbic acid, $\mathrm{pH}$, weight loss, water and solids gain were evaluated; the degree of acceptance by sensory evaluation was also evaluated. The treatment with sucrose (70 ${ }^{\circ}$ Brix and $\left.50{ }^{\circ} \mathrm{C}\right)(\mathrm{T} 3)$ had greater acceptance, with a weight loss of $29.70 \%$, a water loss of $34.4 \%$, a solids gain of $6.83 \%$ and an ascorbic acid content of $11.3 \mathrm{mg} / 100 \mathrm{~g}$. The highest solids gain was obtained with honey at $70^{\circ} \mathrm{Brix}$ and $60^{\circ} \mathrm{C}$; the highest weight and water loss was obtained with honey $\left(60^{\circ}\right.$ Brix and $\left.50^{\circ} \mathrm{C}\right)$ and honey $\left(70^{\circ} \mathrm{Brix}\right.$ and $50^{\circ} \mathrm{C}$ ) respectively.
\end{abstract}

Keywords: osmodeshydration, Vaccinium myrtillus, sucrose, honey of bee.

\footnotetext{
${ }^{1}$ Ingeniero Agroindustrial. Universidad Nacional Toribio Rodríguez de Mendoza de Amazonas.

${ }^{2}$ Ingeniero Agroindustrial. Universidad Nacional Toribio Rodríguez de Mendoza de Amazonas.

$3 *$ Ingeniero Agroindustrial. Universidad Nacional Toribio Rodríguez de Mendoza de Amazonas. Correo electrónico:

sv.olivares@gmail.com
} 


\section{INTRODUCCIÓN}

La deshidratación osmótica (DO), consiste en la remoción del contenido de agua de un alimento, con un aumento simultáneo de sólidos por efecto de la presión osmótica, que ocurre por inmersión de un sólido (entero o en trozos) en una solución hipertónica de uno o más solutos (agente deshidratante) por un cierto tiempo y temperatura específicos (Zapata y Castro, 1999). Además de los flujos de salida de agua y entrada de solutos en el alimento, se observa salida de solutos de bajo peso molecular del propio producto (azúcares, ácidos orgánicos, sales y vitaminas), que ocurre en cantidades mínimas, pero ejerce una importante influencia con relación a la composición y calidad del producto final (Raoult y Wack, 1994).

La calidad del osmodeshidratado, depende en gran mediad del agente empleado y de los factores de proceso, los más importantes son la concentración y la temperatura (Zuluaga, Cortes-Rodríguez, Rodríguez-Sandoval, 2010).

Se han evaluado los procesos de deshidratación osmótica en diversos vegetales y estandarizado los mismos, Zapata y Castro (1999), reportaron trabajos en piña, banano, mandarina, mora, curuba, breva, guayaba, tomate, pimentón, cebolla, mango, aguaymanto, manzana y otros. Asimismo, se ha evaluado diversos agentes osmodeshidratantes como sacarosa, miel de abejas, jarabes azúcar-almidón, azúcar invertido, cloruro de calcio, panela etc. (García, Muñiz, Hernández, Gonzáles y Fernández, 2013).

El arándano (Vaccinium myrtillus), es una fruta, catalogada dentro del grupo de los berries. El cultivo de esta fruta, debido al incremento de su demanda, se viene promoviendo en diversas partes del Perú. En Tío Pucro, Magdalena, Chachapoyas, Amazonas, se produce arándano entre los meses de octubre, a diciembre (Sierra Exportadora, 2015).

Por lo que el objetivo de investigación fue evaluar la temperatura y la concentración de dos agentes osmodeshidratantes en la obtención de arándano deshidratado.

\section{MATERIAL Y MÉTODOS}

El experimento, se realizó en el laboratorio de Ingeniería y Tecnología Agroindustrial de la Universidad Nacional Toribio Rodríguez de Mendoza de Amazonas.

Se emplearon frutos de $V$. myrtillus con madurez fisiológica de 33,44 , sólidos solubles totales $10,7^{\circ}$ Brix, acidez total $0,32 \%$ y pH 5,04, obtenidos en el distrito de Magdalena, provincia de Chachapoyas, región Amazonas.

El procedimiento experimental, se describe en la Figura 1. Se evaluó los agentes osmodeshidratantes, jarabe de sacarosa y miel de abeja a 60 y $70^{\circ}$ Brix con con temperaturas de 50 y $60^{\circ} \mathrm{C}$, bajo un diseño completo al azar (DCA) mostrado en la Tabla 1. Los tratamientos fueron comparados velocidades de pérdida de peso (WR), pérdida de agua (WL) y ganancia de sólidos (SG) (López y Morales, 1998), para lo que se utilizó las fórmulas:

$W R=\frac{(M o-M f)}{M o} \times 100$

Donde: $\mathrm{WR}=$ pérdida de peso, $\mathrm{Mo}=$ peso inicial de la fruta $(\mathrm{g})$ y Mf = peso final de la fruta $(\mathrm{g})$.

$$
W L=\frac{(M o x H o)-(M f x H f)}{M o} \times 100
$$

Donde: $\mathrm{WL}=$ pérdida de agua, $\mathrm{Mo}=$ peso inicial de la fruta $(\mathrm{g}), \mathrm{Mf}=$ peso final de la fruta $(\mathrm{g})$, Ho = humedad inicial de la fruta, como fracción $(\mathrm{mL} / \mathrm{g})$ y $\mathrm{Hf}=$ humedad final de la fruta, como fracción $(\mathrm{mL} / \mathrm{g})$.

$S G=\frac{(M f \times S f)-(\text { Mo } x \text { So })}{M o} \times 100$

Donde: $\mathrm{SG}=$ ganancia de sólidos, $\mathrm{Mo}=$ peso inicial de la fruta $(\mathrm{g}), \mathrm{Mf}=$ peso final de la fruta $(\mathrm{g})$, So = sólidos iniciales y $\mathrm{Sf}=$ sólidos finales.

También, se determinó ${ }^{\circ}$ Brix (refractométrico), $\mathrm{pH}$ [método potenciómetrico; Madrid (1994)], acidez total [por titulación; Madrid (1994)], índice de madurez [ ${ }^{\circ}$ Brix / \% acidez; Primo (1998)], humedad [método 394.06 A.O.A.C., (2000)], cenizas [método de calcinación; A.O.A.C. (1981)] y ácido ascórbico (por titulación). La aceptación sensorial, mediante los aspectos flavor y textura, mediante un DCA con 15 panelistas semientrenados, empleando una escala hedónica de 7 puntos (7: me gusta muchísimo, 6: me gusta, 5: me gusta un poco, 4: no me gusta ni me disgusta, 3: me disgusta un poco, 2: me disgusta, 1: me disgusta muchísimo). Los datos obtenidos fueron sometidos a pruebas de análisis de varianza y comparación de medias de Tukey con 0,05 de significación para evaluar las diferencias de medias. Al mejor tratamiento obtenido se realizó el análisis fisicoquímico con los mismos métodos que se emplearon para la materia prima. 
Tabla 1. Relación de los tratamientos en la osmodeshidratacion y secado en la obtención de arándano deshidratado

\begin{tabular}{|c|c|c|c|c|}
\hline \multirow[b]{2}{*}{ Trat. } & \multicolumn{3}{|c|}{ Osmodeshidratación } & \multirow{2}{*}{$\begin{array}{c}\text { Secado } \\
T^{1}\left({ }^{\circ} \mathrm{C}\right)\end{array}$} \\
\hline & $\begin{array}{l}\text { Agente } \\
\text { osmótico }\end{array}$ & $\begin{array}{l}\text { Jarabe } \\
\text { ('Brix) }\end{array}$ & $\begin{array}{r}T^{1} \\
\left({ }^{\circ} \mathbf{C}\right)\end{array}$ & \\
\hline T1 & Sacarosa & 60 & 50 & 60 \\
\hline T2 & Sacarosa & 60 & 60 & 60 \\
\hline T3 & Sacarosa & 70 & 50 & 60 \\
\hline T4 & Sacarosa & 70 & 60 & 60 \\
\hline T5 & Miel de abeja & 60 & 50 & 60 \\
\hline T6 & Miel de abeja & 60 & 60 & 60 \\
\hline T7 & Miel de abeja & 70 & 50 & 60 \\
\hline T8 & Miel de abeja & 70 & 60 & 60 \\
\hline
\end{tabular}

El proceso de osmodeshidratación se realizó con las condiciones establecidas en el cuadro anterior, pero también es importante mencionar que el tiempo fue constante para los ocho tratamientos y de 20 horas

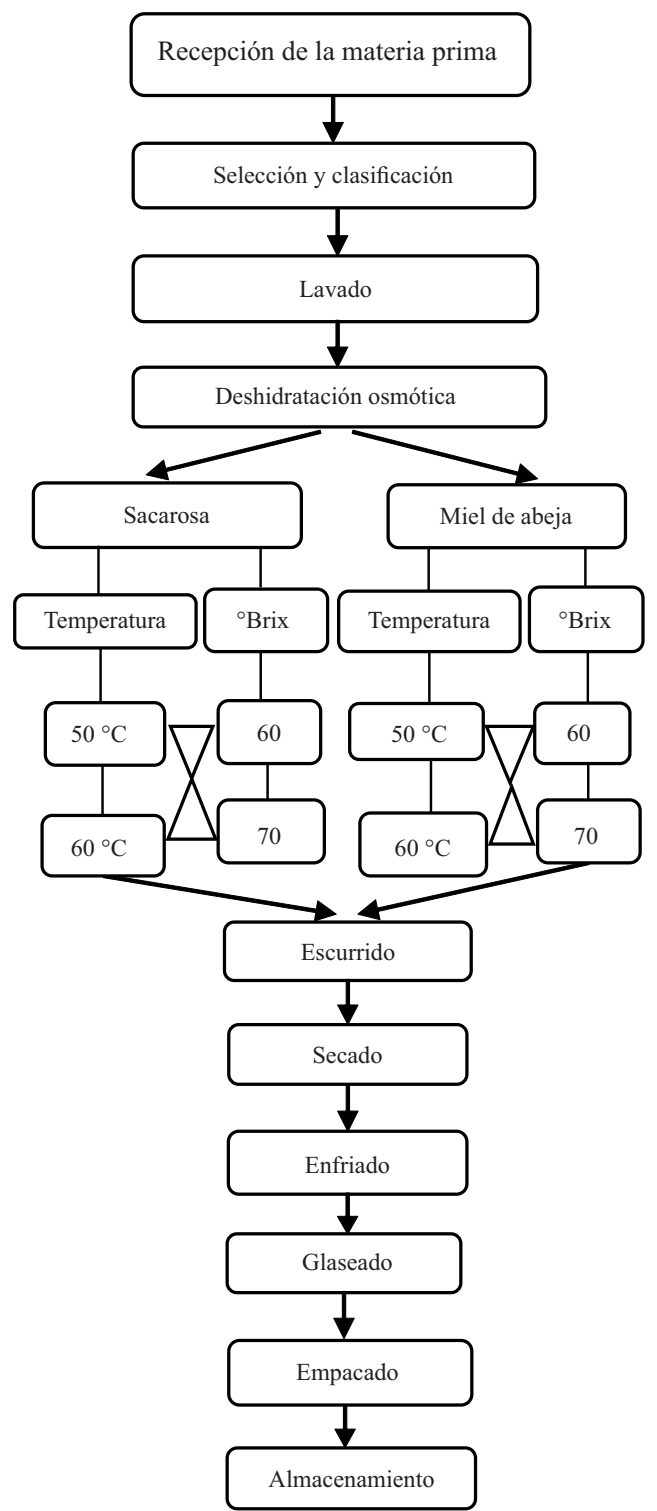

Figura 1. Flujo para la deshidratación osmótica de V. myrtillus

\section{RESULTADOS}

Tabla 2. Valores de las características fisicoquímicas del arándano fresco

\begin{tabular}{lc}
\hline \multicolumn{1}{c}{$\begin{array}{c}\text { Características fisicoquímicas } \\
\text { del Arándano }\end{array}$} & Valor \\
\hline Acidez total $^{1}(\%)$ & 0,32 \\
Sólidos Solubles ( ${ }^{\circ}$ Brix) & 10,70 \\
Índice de madurez & 33,44 \\
Humedad (\%) & 83,10 \\
Cenizas (\%) & 0,21 \\
pH & 5,04 \\
Ácido ascórbico (mg) & 13,00 \\
\hline \multicolumn{1}{c}{ (1) ácido ascórbico equivalente. }
\end{tabular}

La fruta utilizada tuvo una acidez total de $0,32 \%$ y un índice de madurez de 33,44 una elevada humedad (83\%) y pH de 5,04.

Los tratamientos (fruta deshidratada) perdieron entre 28 y $32 \%$ de peso, y no difieren mucho entre el agente osmodeshidratante. La pérdida de peso está directamente correlacionado con la perdida de agua de la frutas, tal como se evidencia en la y se evidenció una ganancia de peso entre 6 y $11 \%$ (Figura 2).

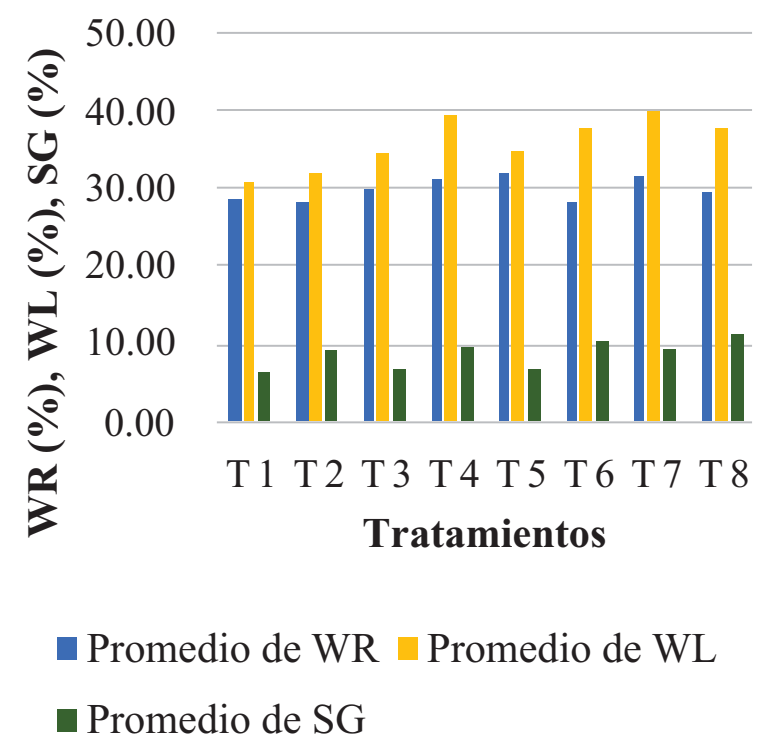

Figura 2. Porcentaje de pérdida de peso (WR), pérdida de agua (WL) y ganancia de sólidos ( S G ), durante la osmodeshidratación de arándano 
Tabla 3. Valor acumulado en los parámetros (Aspecto, flavor y textura), de las características organolépticas de arándano osmodeshidratado

\begin{tabular}{|c|c|c|c|c|}
\hline \multirow{2}{*}{ 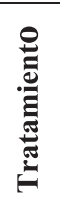 } & \multicolumn{3}{|c|}{ Características Organolépticas } & \multirow[t]{2}{*}{ Total } \\
\hline & Aspecto & Flavor & Textura & \\
\hline T1 & 59 & 60 & 58 & 177 \\
\hline $\mathbf{T} 2$ & 73 & 69 & 72 & 214 \\
\hline T3 & 83 & 76 & 78 & 237 \\
\hline $\mathbf{T 4}$ & 72 & 73 & 68 & 213 \\
\hline T5 & 61 & 62 & 57 & 180 \\
\hline T6 & 73 & 75 & 67 & 215 \\
\hline T7 & 76 & 75 & 82 & 233 \\
\hline T8 & 70 & 67 & 60 & 197 \\
\hline
\end{tabular}

El tratamiento con mejores resultados fue $\mathrm{T} 3$ (sacarosa con $70{ }^{\circ}$ Brix y $60^{\circ} \mathrm{C}$ ); obtuvo mayor puntuación en aspecto, flavor y en textura; sin embargo en este último aspecto, fue superado por el T7 (Tabla 3).

Tabla 4. Caracterización fisicoquímica del mejor tratamiento (T3)

\begin{tabular}{|c|c|}
\hline $\begin{array}{c}\text { Características fisicoquímicas } \\
\text { del Arándano }\end{array}$ & Valor \\
\hline $\operatorname{Acidez}_{\text {total }}{ }^{1}(\%)$ & 0.37 \\
\hline Sólidos Solubles ( ${ }^{\circ}$ Brix) & 25 \\
\hline Humedad (\%) & 69.31 \\
\hline Cenizas (\%) & 0.26 \\
\hline $\mathrm{pH}$ & 5 \\
\hline Ácido ascórbico (mg) & 11.3 \\
\hline
\end{tabular}

\section{DISCUSIÓN}

La mayor pérdida de agua (WL) se obtuvo en el con los tratamientos que mayor concentración de jarabe tuvieron (70 ante $\left.60^{\circ} \mathrm{Brix}\right)$. Tal como señala Conway, et al. (1983), además del tiempo de inmersión, la concentración del osmodeshidratante es directamente proporcional a la cantidad de agua perdida, porque el incremento en los niveles sacarosa causa un aumento en la fuerza impulsora que favorece la pérdida de agua (Lenart, et al., 1984). Además, debe tenerse en cuenta el tiempo de inmersión, puesto que el tiempo de equilibrio osmótico depende de la fruta a deshidratar (Lazaridez, 1995); resultados similares han sido encontrados por Mazzeo et al. (2006), Barrera y Pillman (2010) y Arista y Cruz (2014) cuando deshidrataron otro tipo de frutas.

El proceso influye en la pérdida de ácido ascórbico, puesto que debido a su naturaleza hidrosoluble, éste se pierde por lixiviación (Hernandez y Sastre, 1999).

En general, todos los tratamientos obtuvieron un una elevada puntuación en cuanto a la aceptación; concordando con Ordoñez y Lopez (2002), quienes afirman que estos procesos mejoran las características organolépticas de las frutas.

La sacarosa presentó mejor capacidad osmótica ante miel de abeja, concordando con Sharma (2003) y el mejor tratamiento podría tener buena aceptación en el mercado ya obtuvo buenos resultados de aceptabilidad y buenas características fisicoquímicas (Carpenter, 2002).

\section{CONCLUSIONES}

Los parámetros de osmodeshidratación más adecuados para arándanos son la utilización de jarabe de sacarosa a $70^{\circ}$ Brix, temperatura de inmersión $50^{\circ} \mathrm{C}$, temperatura de secado en secador de bandejas $60^{\circ} \mathrm{C}$ y velocidad de aire a $3,5 \mathrm{~m} / \mathrm{s}$.

\section{REFERENCIAS BIBLIOGRÁFICAS}

Arista, L., y Cruz, O. (2014). Efecto de la velocidad de agitación magnética sobre la deshidratación osmótica de cocona (Solanum sessiliflorum) en soluciones de sacarosa y miel de abeja (Tesis de grado). Universidad Nacional Toribio Rodríguez de Mendoza de Amazonas, Perú.

Carpenter, R. (2002). Análisis Sensorial en el Desarrollo y Control de Calidad de Alimentos. Zaragosa - España. Editorial Acribia S. A.

Conway, J., Castaigne, G., Picard, G., y Vovan, X. (1983). Mass transfer consideratios in the 
osmoticdehydration of apples. Canadian Institute Food Science and Technology Journal, 16(1), 25-29.

García, A., Muñiz, S., Hernández, A., González, L. M., y Fernández, D. (2013). Análisis comparativo de la cinética de deshidratación osmótica y por flujo de Aire Caliente de la Piña (Ananas Comosus, variedad Cayena lisa). Revista Ciencias Técnicas Agropecuarias, 22(1), 62-69.

Hernandez, M., y Sastre, A. (1999). Tratado de nutrición. Ediciones Diaz de Santos S.A. Madrid-España.

Lenart, A., y Flink, J. M. (1984). Osmotic concentration of potato:I Criteria for the end - point of the osmosis process. J. of Food Process Engineering.

López, B., y Morales, J. (1998). Deshidratación osmótica de la mora. Conferencia del segundo seminario de frutales de clima frío. Manizales, Colombia. Agosto 12-14.

Mazzeo, M., Leon, L., Hernandez, H., \& Guapacha, H. (2006). Deshidratación osmótica de arveja (Pisum sativum L.) y habichuela (Phaesehaseolus vulgaris L.) utilizando soluciones de glicerol y cloruro de sodio. Revista Vector, 1(1), 9-28.

Ordoñez, H., y López, B. (2002). Efecto de la presión y temperatura en la elaboración de hojuelas de manzana variedad Anna. Noos., 15, 85 99.

Raoult-Wack, A. L. (1994). Recent advances in the osmotic dehydration of foods. Trends in Food Science \& Technology, 5(8), 255-260.

Sharma, S. (2003). Ingeniería de alimentos. Operaciones Unitarias Prácticas de Laboratorio. liwey: Limusa. 2003, 225235 .

Zapata, J. E., y Castro, G. (1999). Deshidratación osmótica de frutas y vegetales. Revista Facultad Nacional de Agronomía, 52(1), 451-466.

Zuluaga, J. D., Cortes-Rodríguez, M, y RodríguezSandoval, E. (2010). Evaluación de las características físicas de mango deshidratado aplicando secado por aire caliente y deshidratación osmótica. Revista de la Facultad de Ingeniería, 25(4), 127135. 